\title{
PRC1 coordinates timing of sexual differentiation of female primordial germ cells
}

Shihori Yokobayashi ${ }^{1,}$, Ching-Yeu Liang ${ }^{1,2}$, Hubertus Kohler ${ }^{1}$, Peter Nestorov ${ }^{1,2}$, Zichuan Liu ${ }^{1}$, Miguel Vidal ${ }^{3}$, Maarten van Lohuizen ${ }^{4}$, Tim C. Roloff ${ }^{1}$ and Antoine H.F.M. Peters $^{1,2}$

1. Friedrich Miescher Institute for Biomedical Research (FMI), Maulbeerstrasse 66, CH4058 Basel, Switzerland

2. Faculty of Sciences, University of Basel, $\mathrm{CH}-4056$ Basel, Switzerland

3. Centro de Investigaciones Biológicas, Consejo Superior de Investigaciones Científicas (CSIC), 28040 Madrid, Spain

4. Division of Molecular Genetics and Centre for Biomedical Genetics, the Netherlands Cancer Institute (NKI), 1066 CX Amsterdam, the Netherlands

*. Present address: Department of Reprogramming Science, Center for iPS Cell Research and Application, Kyoto University, 53 Kawahara-cho, Shogoin Yoshida, Sakyo-ku, Kyoto 606-8507, Japan

Corresponding author:

Antoine Peters

Phone: $\quad+41616978761$

Fax: $\quad+41616973976$

Email: $\quad$ antoine.peters@fmi.ch

Keywords: 
Polycomb Repressive Complex 1, primordial germ cells, sex differentiation, meiosis, retinoic acid signaling 
In mammals, sex differentiation of primordial germ cells (PGCs) is determined by extrinsic cues from the environment ${ }^{1}$. In female PGCs, expression of Stimulated by retinoic acid 8 (Stra8) and meiosis are induced in response to retinoic acid (RA) provided from the mesonephroi ${ }^{2-5}$. Given the widespread role of RA signaling during development ${ }^{6,7}$, the molecular mechanism specifying the competence of PGCs to timely express Stra8 and enter meiosis are unknown ${ }^{2,8}$. Here we identify gene dosage dependent roles in PGC development for Ring1 and Rnf2, two central components of the Polycomb Repressive Complex 1 (PRC1 $^{9,10}$. Both paralogs are essential for PGC development between day 10.5 and 11.5 of gestation. Rnf2 is subsequently required in female PGCs to maintain high levels of Oct4 and Nanog expression ${ }^{11}$, and to prevent premature induction of meiotic gene expression and entry into meiotic prophase. Chemical inhibition of RA signaling partially suppresses precocious Oct4 down-regulation and Stra8 activation in Rnf2-deficient female PGCs. Chromatin immunoprecipitation analyses show that Stra8 is a direct target of PRC1 and PRC2 in PGCs. These data demonstrate the importance of PRC1 gene dosage in PGC development and in coordinating the timing of sex differentiation of female PGCs by antagonizing extrinsic RA signaling.

In mammalian somatic cells, PRC1 and PRC2 proteins are transcriptional repressors that function in large multi-protein complexes and that modify chromatin by monoubiquitinating histone $\mathrm{H} 2 \mathrm{~A}$ at lysine 119 (H2AK119u1) and tri-methylating $\mathrm{H} 3$ at lysine 27 (H3K27me3), respectively ${ }^{9,12}$. At day 12.5 of embryonic development (E12.5), we observed in PGCs marked by Cdh1 (E-cadherin) staining ${ }^{13}$ nuclear localization of PRC1 components Rnf2/Ring1b, Mel18/Pcgf2 and Rybp (Fig. 1a; Supplementary Fig. 1) as well as a robust $\mathrm{H} 2 \mathrm{AK} 119 \mathrm{u} 1$ signal suggesting the presence of catalytically active PRC1 complexes (Fig. 1a). To address the function of PRC1 in PGC development (Supplementary Fig. 2), we conditionally deleted Rnf2 in PGCs from E9.5 onwards by 
generating mice carrying a floxed and a mutant allele of $R n f 2\left(R n f 2^{F / \Delta}\right)$ and a Cre recombinase driven by the Tnap promoter (Tnap-cre) $)^{14,15}$. To concomitantly assess possible functional redundancy with the Rnf2 paralog $\operatorname{Ring} 1^{10,16}$, we studied mice that were either heterozygous or homozygous deficient for Ring $1^{17}$, further referred to as $R n f 2^{c k o}$ and $R i n g 1^{\Delta} / R n f 2^{c k o}$ respectively (Supplementary Fig. 3, 6a). This strategy resulted in $\sim 90 \%$ deletion efficiency at E11.5 (Supplementary Fig. 4). At E12.5, Rnf2, Mel18, Rybp and H2AK119u1 were lost in PGCs of Rnf2 ${ }^{\text {cko }}$ but not Rnf2 ${ }^{F / \Delta}$ embryos indicating that complex stability and catalytic activity of PRC1 is regulated by Rnf2 in PGCs at E12.5 (Fig. 1a; Supplementary Fig. 4). In contrast, Ezh2 and H3K27me3 levels were similar in Rnf2 ${ }^{\text {cko }}$ versus control PGCs suggesting globally unaltered PRC2 function in Rnf2 $2^{\text {cko }}$ PGCs (Supplementary Fig. 5).

To study the fate of Rnf2-deficient PGCs, we analyzed expression of the pluripotency and germ cell marker Oct4 in whole gonads of E10.5 to E13.5 embryos (Fig. 1b; Supplementary Fig. 2). We observed a strong reduction of Oct4-positive PGCs specifically in female Rnf2 ${ }^{\text {cko }}$ embryos but not in male $R n f 2^{\text {cko }}$ or control embryos, starting around E12.5 of gestation (Fig. 1b, c). In contrast, double deficiency of Ring1 and Rnf2 caused a strong reduction of Oct4-positive PGCs already at E11.5 in both sexes (Supplementary Fig. 6b, c), indicating an essential role for PRC1 in PGCs after their migration into the embryonic gonad (Supplementary Fig. 2) ${ }^{11}$.

To further dissect the role of Rnf2 in regulating Oct4 expression versus PGC development, we assessed in Rnf2 ${ }^{\text {cko }}$ embryos co-expression of Oct4 and Rnf2 at E12.5 in Cdh1-positive PGCs (Fig. 1d). The number of PGCs lacking detectable Rnf2 protein was strongly reduced in female but not male gonads, despite that gonads of opposite sexes harbored comparable numbers of Rnf2-deficient PGCs at E12.0 (Supplementary Fig. 4a, data not shown). We further observed a pronounced downregulation of Oct4 protein in female and some male Rnf2-deficient PGCs (Fig. 1d). These data indicate that Rnf2 contributes to maintaining Oct4 expression particularly in female PGCs beginning between E12.0 and E12.5. 
We subsequently investigated the mechanism underlying the reduction in female Rnf2-deficient PGCs. We failed to observe increased levels of apoptosis or major changes in cell cycle progression (data not shown). To study changes in gene expression, we introduced an Oct4( $\triangle P E)$-GFP transgene ${ }^{18}$ into the Rnf2 ${ }^{\text {cko }}$ strain and isolated pure populations of PGCs by Fluorescent Activated Cell Sorting (FACS) (Fig. 1e). By RT-qPCR, we barely detected Rnf2 transcripts in isolated Rnf2 ${ }^{\text {cko }}$ PGCs, confirming efficient deletion of the $R n f 2^{F}$ allele by Tnap-Cre (Fig. 1f; see also Supplementary Fig. 4). Hence, we subsequently refer to PGCs from Rnf2 ${ }^{\text {cko }}$ embryos as $R n f 2^{\Lambda}$. We also noticed significant reductions in GFP intensities in $R n f 2^{\Delta}$ PGCs isolated from female and male E11.5 and E12.5 embryos compared to controls (Fig. 1e), suggesting decreased $\operatorname{Oct}(\triangle P E)$ promoter activity in $R_{n f 2^{\Delta}}$ PGCs. RT-qPCR analysis showed significantly reduced Oct4 and Nanog expression in female GFPpositive Rnf2 $^{\perp}$ PGCs at E11.5 and E12.5 (Fig. 1f; data not shown). Thus, Rnf2 is required for maintaining expression of pluripotency factors in PGCs.

We then performed genome-wide expression analysis on purified PGCs. Twelve-fold more genes were mis-regulated in female than male Rnf2 ${ }^{\Delta}$ PGCs at E12.5 (Fig. 2a; Supplementary Table1). Consistent with PRC1 being a transcriptional repressor, $\sim 90 \%$ of mis-expressed genes were up-regulated. At E11.5, we only observed a few mis-regulated genes in Rnf2 ${ }^{\Delta}$ PGCs (Supplementary Table 1), consistent with the timing of the mutant phenotype. According to Gene Ontology analysis, gene functions related to meiosis (synapsis, sister chromatid cohesion) were highly over-represented among genes up-regulated in female $\operatorname{Rnf}^{\Lambda}{ }^{\wedge G C s}$. In contrast, nucleosome functions were enriched in down-regulated genes, as reported previously for Ring1 and Rnf2 double deficient Germinal Vesicle oocytes ${ }^{10}$ (Supplementary Fig. 7; Supplementary Table 2). Surprisingly, we did not find a significant over-representation of developmental gene functions in $R n f 2^{\perp}$ PGCs, a classical feature of PRC1 deficiency in ESCs ${ }^{16,19}$ and GV oocytes ${ }^{10}$. To test whether this is due to functional redundancy of the Ring1 paralog, we profiled expression in $R$ ing $1^{\Delta} / R_{n f 2}{ }^{\wedge}$ PGCs purified from E11.5 
embryos. Consistently, many developmental gene functions were over-represented among up-regulated genes (Supplementary Fig. 8; Supplementary Table 2). These data indicate that while Ring1 expression is sufficient to safeguard global repression of canonical PcG target genes in PGCs, Rnf2 is required in female E12.5 PGCs for repression of genes driving meiosis.

At E11.5, gonads still possess the potential to develop either into ovaries or testes while they are committed to a sex-specific differentiation process two days later. To relate aberrant expression in $\mathrm{Rnf2}^{\wedge} \mathrm{PGCs}$ to changes occurring during normal PGC differentiation, we profiled expression in Rnf2 $^{+}$PGCs isolated between E11.5 and E13.5. In this developmental period, 6-fold more genes were up-regulated in female (810) than male (132) Rnf2 ${ }^{+}$PGCs, with an additional 196 genes being up-regulated in both sexes, suggesting the activation of female- and male-specific "PGC Differentiation Programs" (PDPs) (Fig. 2b). Among genes up-regulated in E12.5 female Rnf2 ${ }^{\wedge}$ PGCs, $223(43 \%)$ were part of the female PDP (Fig. 2b; Supplementary Fig. 7b) ( $P$-value 2.67E-159; geometric-test), being activated about one day ahead compared to those in Rnf2 ${ }^{+}$PGCs (Fig. 2c).

We next identified that 24 out of 119 genes annotated with the GO-term "meiosis" (GO:0007126) were part of the female PDP, likely reflecting activation of an "Early Meiosis Program” (Fig. 2d). Among these 24 genes, 10 were precociously upregulated in female $\mathrm{Rnf2}^{\wedge} \mathrm{PGCs}$ including Stra8, required for meiotic initiation ${ }^{5}$, and others like Rec8, Sycp3, and Hormad2 executing key functions in cohesion, chromosome synapsis and recombination ${ }^{20-22}$. By RT-qPCR, we measured high Stra8, Rec8 and Sycp3 mRNA levels at E13.5 in female Rnf2 ${ }^{+}$PGCs (Fig. 3a). At E11.5 and E12.5, Stra8 and Rec8 were precociously activated with up to 20-fold higher expression levels in female $R n f 2^{\Lambda}$ versus $R n f 2^{+}$PGCs. Sycp3 was expressed in $R_{n f 2}{ }^{+}$ PGCs from both sexes with up to 4 -fold increased levels in female Rnf2 ${ }^{\Lambda}$ PGCs (Fig. 3a). IF analyses revealed strong Stra8 nuclear localization already at E12.5 in PGCs of $R n f 2^{\text {cko }}$ gonads, whereas the protein only started to accumulate in control PGCs at 
E13.5 (Fig. 3b; Supplementary 7c). For Sycp3, we observed focal nuclear staining at E13.5 and synaptonemal complex staining at E14.5 in Rnf2 $^{\Delta}$ germ cells while only axial elements of meiotic chromosomes were visible in Rnf2 $^{+}$germ cells at E14.5 (Fig. 3c, $3 d$, data not shown). These data indicate that precocious transcriptional activation of Stra8 and other PGC differentiation and meiosis genes in female Rnf2 $^{\perp}$ PGCs induces these cells to prematurely stop proliferation and enter into meiotic prophase, hence accounting for the lower number of female $R n f 2^{\wedge}$ PGCs at E12.5. We measured in female Rnf $^{\wedge}$ PGCs at E11.5 and 12.5 also increased expression of genes functioning in retinoic acid metabolism such as Aldh1a2, Crabp1 and Crabp2 (Supplementary Fig. 7d; data not shown), likely enhancing RA-signaling and meiotic entry in a feed forward manner ${ }^{23}$.

Why is Stra8 transcription abnormally activated only in female Rnf2 ${ }^{\wedge}$ PGCs? In male gonads, RA-mediated induction of Stra8 is counteracted by the somatically expressed retinoid-degrading enzyme Cyp26b1 and by Fgf9 (Supplementary Fig. $2)^{3,4,24}$. PRC1 may therefore not be required to suppress Stra8 expression in males. To study this possibility, we aimed at overcoming the antagonizing activity of Cyp26b1 and cultured E11.5 genital ridges for 24 hrs in the presence of all-trans RA (ATRA) $)^{3,4}$. ATRA increased Stra8 expression in isolated $R n f 2^{+}$PGCs of both sexes to levels comparable to what we measured in female $\operatorname{Rnf}^{\perp}$ PGCs treated with DMSO (Fig. 4a). Intriguingly, ATRA additionally enhanced Stra8 expression in male as in female Rnf2 $^{\perp}$ PGCs to $>5$ fold higher levels compared to Rnf2 ${ }^{+}$PGCs (Fig. 4a), indicating that PRC1 effectively suppresses RA-induced Stra8 activation in PGCs of both sexes. We observed comparable responses for Rec8 and Sycp3 expression (Fig. 4a). In contrast, Stra8 activation was completely suppressed in all genotypes by treatment with WIN18446, an inhibitor of the RA biogenesis pathway ${ }^{25}$ (Fig. 4a). Likewise, two-day in vivo exposure of PGCs to WIN18446 in developing embryos suppressed premature Stra8 protein expression (Supplementary Fig. 9a, 9b). Lastly, numbers of Oct4-expressing PGCs in $R n f 2^{\text {cko }}$ gonads were significantly increased upon treatment in vitro and in vivo with 
WIN18446 (Fig. 4b, Supplementary Fig. 9c, 9d). These results indicate that sensitization of PGCs to RA signaling by Rnf2-deficiency contributes to precocious exit from the PGC state and activation of the meiotic program in female gonads.

Subsequently, the majority of $R n f 2^{\Delta}$ PGCs were unable to complete meiosis and to develop into mature oocytes (Supplementary Fig. 10), possibly as a consequence of the precocious entry into meiosis that may eventually impair, perhaps in concert with other aberrant changes in gene expression (Fig. 2b), the execution of the natural female PDP and oogenesis. Alternatively, PRC1 may exert a separate essential function later during meiotic progression.

Finally, we performed a micro-chromatin immunoprecipitation ( $\mu \mathrm{ChIP})$ assay on isolated Rnf2 ${ }^{+}$PGCs. At E11.5, the Stra8 promoter was strongly enriched with PRC2mediated H3K27me3 and with H3K4me3, indicative of a PcG repressed, yet potentially transcriptionally primed, chromatin state. During subsequent stages, we noticed a progressive decrease in $\mathrm{H} 3 \mathrm{~K} 27 \mathrm{me} 3$ levels in female PGCs consistent with Stra8 transcriptional activation, while the promoter remained bivalent in male PGCs (Fig. 4c). In contrast, the Hoxa9 promoter was bivalent in all conditions (Supplementary Fig. 11). Likewise, we detected Rnf2 association with the Stra8 promoter at E11.5 and E12.5 PGCs and a significant decrease in Rnf2 occupancy in female but not male PGCs at E13.5. Together, these experiments suggest that Stra8 is directly regulated by PRC2 and PRC1 in PGCs of both sexes.

In summary, we identified an essential role for PRC1 in PGC development between E10.5 to E11.5. Later, between E11.5 to E13.5, Rnf2 effectively modulates the sensitivity of PGCs to RA-mediated induction of meiosis by directly controlling the competence of Stra8 and likely of other meiotic genes for transcriptional activation. Like the proposed role of $C b x 2$ in temporal colinearity of $H_{0 x}$ gene activation ${ }^{26}$, we speculate that PRC1 maintains repression of Stra8 and further genes of the PGC differentiation and early meiosis programs until RA signaling has reached a certain threshold (Fig. 4d). In addition, PRC1 regulates expression of pluripotency genes in 
PGCs, possibly indirectly by suppressing transcription of negative regulators. Impairing PRC1 function in these parallel pathways likely leads to a synergistic effect, thereby promoting premature transition from proliferation into meiosis.

\section{METHODS SUMMARY}

Embryos were obtained by timed matings, by scoring noon of the day following mating as embryonic day 0.5 (E0.5). Genital ridges were cultured in drops of Dulbecco's Minimal Eagle Medium (DMEM) supplemented with $10 \%$ fetal calf serum at $37^{\circ} \mathrm{C}$ with $5 \% \mathrm{CO}_{2}$ in air. For expression profiling, we collected in triplicate 500 PGCs per embryo by FACS and processed, hybridized to Affymetrix Mouse Gene 1.0 arrays and analyzed as described previously ${ }^{10}$. GO terms were obtained using GO Stat (http://gostat.wehi.edu.au). $\mu$ ChIP was performed as described previously ${ }^{27}$.

Full methods and associated references are available in the online version of the paper at www.nature.com/nature.

1 Brennan, J. \& Capel, B. One tissue, two fates: molecular genetic events that underlie testis versus ovary development. Nat Rev Genet 5, 509-521 (2004).

2 Menke, D. B., Koubova, J. \& Page, D. C. Sexual differentiation of germ cells in XX mouse gonads occurs in an anterior-to-posterior wave. Dev Biol 262, 303-312 (2003).

3 Koubova, J. et al. Retinoic acid regulates sex-specific timing of meiotic initiation in mice. Proc Natl Acad Sci U S A 103, 2474-2479 (2006).

4 Bowles, J. et al. Retinoid signaling determines germ cell fate in mice. Science 312, 596-600 (2006).

5 Baltus, A. E. et al. In germ cells of mouse embryonic ovaries, the decision to enter meiosis precedes premeiotic DNA replication. Nat Genet 38, 1430-1434 (2006). 
6 Deschamps, J. Ancestral and recently recruited global control of the Hox genes in development. Curr Opin Genet Dev 17, 422-427 (2007).

7 Duester, G. Retinoic acid synthesis and signaling during early organogenesis. Cell 134, 921-931 (2008).

8 Oulad-Abdelghani, M. et al. Characterization of a premeiotic germ cell-specific cytoplasmic protein encoded by Stra8, a novel retinoic acid-responsive gene. J Cell Biol 135, 469-477 (1996).

9 Sparmann, A. \& van Lohuizen, M. Polycomb silencers control cell fate, development and cancer. Nat Rev Cancer 6, 846-856 (2006).

10 Posfai, E. et al. Polycomb function during oogenesis is required for mouse embryonic development. Genes Dev (2012).

11 Saitou, M., Kagiwada, S. \& Kurimoto, K. Epigenetic reprogramming in mouse pre-implantation development and primordial germ cells. Development 139, 15-31 (2012).

12 Simon, J. A. \& Kingston, R. E. Mechanisms of polycomb gene silencing: knowns and unknowns. Nat Rev Mol Cell Biol 10, 697-708 (2009).

13 Di Carlo, A. \& De Felici, M. A role for E-cadherin in mouse primordial germ cell development. Developmental biology 226, 209-219 (2000).

14 Puschendorf, M. et al. PRC1 and Suv39h specify parental asymmetry at constitutive heterochromatin in early mouse embryos. Nat Genet 40, 411-420 (2008).

15 Lomeli, H., Ramos-Mejia, V., Gertsenstein, M., Lobe, C. G. \& Nagy, A. Targeted insertion of Cre recombinase into the TNAP gene: excision in primordial germ cells. Genesis 26, 116-117 (2000).

16 Endoh, M. et al. Polycomb group proteins Ring1A/B are functionally linked to the core transcriptional regulatory circuitry to maintain ES cell identity. Development $135,1513-1524$ (2008).

17 del Mar Lorente, M. et al. Loss- and gain-of-function mutations show a polycomb group function for Ring1A in mice. Development 127, 5093-5100 (2000). 
18 Yoshimizu, T. et al. Germline-specific expression of the Oct-4/green fluorescent protein (GFP) transgene in mice. Dev Growth Differ 41, 675-684 (1999).

19 Leeb, M. et al. Polycomb complexes act redundantly to repress genomic repeats and genes. Genes Dev 24, 265-276 (2010).

20 Xu, H., Beasley, M. D., Warren, W. D., van der Horst, G. T. \& McKay, M. J. Absence of mouse REC8 cohesin promotes synapsis of sister chromatids in meiosis. Developmental cell 8, 949-961 (2005).

21 Yuan, L. et al. Female germ cell aneuploidy and embryo death in mice lacking the meiosis-specific protein SCP3. Science 296, 1115-1118 (2002).

22 Wojtasz, L. et al. Meiotic DNA double-strand breaks and chromosome asynapsis in mice are monitored by distinct HORMAD2-independent and -dependent mechanisms. Genes \& development 26, 958-973 (2012).

23 Maden, M. Retinoic acid in the development, regeneration and maintenance of the nervous system. Nat Rev Neurosci 8, 755-765 (2007).

24 Bowles, J. et al. FGF9 suppresses meiosis and promotes male germ cell fate in mice. Dev Cell 19, 440-449 (2010).

25 Hogarth, C. A. et al. Suppression of Stra8 expression in the mouse gonad by WIN 18,446. Biol Reprod 84, 957-965 (2011).

26 Bel-Vialar, S. et al. Altered retinoic acid sensitivity and temporal expression of Hox genes in polycomb-M33-deficient mice. Dev Biol 224, 238-249 (2000).

27 Dahl, J. A. \& Collas, P. A rapid micro chromatin immunoprecipitation assay (microChIP). Nat Protoc 3, 1032-1045 (2008).

Supplementary Information is available in the online version of the paper.

Acknowledgements We gratefully thank Dr. Nagy and Dr. Schöler for the Tnap-cre and Oct4( $\triangle P E)$-GFP mice respectively, and Dr. Michael Griswold for the Stra8 
antibody. We are grateful to Laurent Gelman (microscopy \& imaging), Lukas Burger, Michael Stadler (bioinformatics), Erik Cabuy, Stephane Thiry, Kirsten Jacobeit (functional genomics) and the FMI animal facility for excellent assistance. We thank members of the Peters laboratory particularly Mathieu Tardat, Serap Erkek and Mark Gill for experimental support and discussions.

S.Y. is a recipient of a HFSP long-term fellowship and a JSPS postdoctoral fellowship. Research in the Peters lab is supported by the Novartis Research Foundation, the Swiss National Science Foundation (31003A_125386 and NRP 63 - Stem Cells and Regenerative Medicine), SystemsX.ch (Cell plasticity), the Japanese Swiss Science and Technology Cooperation Program, the European Network of Excellence "The Epigenome" and the EMBO YIP program.

Author Contributions S.Y. and A.H.F.M.P. conceived and designed the experiments. S.Y. performed almost all experiments. C.-Y.L. performed $\mu$ ChIP experiments. H.K. performed FACS isolations. Z.L. isolated GV oocytes. P.N. isolated RNA for RNAsequencing and provided advice on START-qPCR. M.V. provided Ring1 deficient mice. M.v.L. provided conditionally deficient Rnf2 mice and antibodies. T.C.R. assisted in microarray and RNA-sequencing analysis. S.Y., C.-Y.L and A.H.F.M.P. analyzed the data. S.Y. and A.H.F.M.P. wrote the manuscript.

Author Information Micro-array and RNA-sequencing data have been deposited in the Gene Expression Omnibus under accession numbers GSE42782 and GSE42852 respectively. Reprints and permissions information is available at www.nature.com/reprints. The authors declare no competing financial interests. Correspondence and requests for materials should be addressed to A.H.F.M.P. (antoine.peters@fmi.ch).

\section{Figure legends}


Figure 1 | Rnf2 regulates PGC development and Oct4 and Nanog expression in $\boldsymbol{R n f 2}^{\text {cko }}$ female gonads. a, IF staining of H2AK119u1, Rnf2 and Cdh1 with DAPI in $R n f 2^{+}$and $R n f 2^{c k o}$ gonadal sections from E12.5 female embryos. Arrowheads: Rnf2 ${ }^{+}$ PGCs; asterisks: Rnf2 ${ }^{\perp}$ PGCs. Scale bars: $10 \mu \mathrm{m}$. b, IF staining of Oct4 in E13.5 Rnf2 ${ }^{+}$ and $R n f 2^{\text {cko }}$ whole gonads and mesonephroi. Scale bars: $300 \mu \mathrm{m}$. c, Average number of Oct4-positive cells in whole gonads at E10.5 to E13.5. Error bars indicate +s.d.. $\mathrm{n}=2$ 12. ${ }^{*} P<0.005 ;{ }^{* *} P<1.0 \mathrm{E}-05$ (t-test). d, Classification of Cdh1-positive PGCs according to Rnf2 and Oct4 protein levels in $R n f 2^{+}$and $R n f 2^{\text {cko }}$ E12.5 gonads. Y-axis represents the number of PGCs that was normalized to areas analyzed $\left(10,000 \mu \mathrm{m}^{2}\right)$. In brackets: number of PGCs scored per embryo. ${ }^{*} P<1.0 \mathrm{E}-08$ (chi-squared test). e, Representative histograms showing Oct4( $\triangle P E)$-GFP signals in PGCs from female $R_{n f 2}{ }^{+}$and $R_{n f 2}{ }^{c k o}$ E12.5 gonads. Boxplots showing the ratios of PGCs with high GFP intensity ( $>10^{3}$, enclosed by dashed line in histogram) over all GFP-positive cells (enclosed by solid line) in different embryos. In brackets: numbers of embryos analyzed. ${ }^{*} P<0.05 ;{ }^{*} P<0.005$ (t-test). f, Representative RT-qPCR data of Rnf2, Oct4 and Nanog in $\mathrm{Rnf2}^{+}$and $\mathrm{Rnf}^{\Delta}{ }^{\mathrm{P}}$ PCs (normalized to Tbp). Error bars indicate +s.d. of 2-3 technical replicates. ${ }^{*} P<0.05 ;{ }^{* *} P<0.01$ (t-test).

Figure 2 | Rnf2 deficiency induces extensive transcriptional mis-regulation in female PGCs. a, Venn diagrams showing numbers of genes mis-regulated in male and female Rnf2 ${ }^{\wedge}$ PGCs compared to Rnf2 ${ }^{+}$PGCs at E12.5. b, Venn diagram showing numbers of genes up-regulated in control female (red) and male (blue) PGCs between E11.5 and E13.5 and in female Rnf2 ${ }^{\wedge}$ PGCs compared to Rnf2 ${ }^{+}$PGCs at E12.5 (pink oval). c, Relative expression levels of probe sets up-regulated in female and male Rnf2 $^{+}$PGCs between E11.5 and E13.5 in various samples indicated. Unsupervised clustering analysis shows clustering of female E12.5 Rnf2 ${ }^{\wedge}$ PGCs with female E13.5 $R_{n f 2}{ }^{+}$PGCs while E12.5 male $R_{n f 2}{ }^{\Lambda}$ and $R_{n f 2}{ }^{+}$PGCs clustered together. d, Micro- 
array expression values of Early Meiosis Program genes. Genes significantly upregulated in $R n f 2^{\Delta}$ PGCs of both sexes are indicated in bold; those in female $\operatorname{Rnf2}^{\Delta}$ PGCs only in bold-star. $\mathrm{n}=3$ per condition; fold change $>1.5$; adjusted $P$-value $<0.05$.

Figure $3 \mid$ Female $\operatorname{Rnf2}^{\perp}$ PGCs enter precociously into meiotic prophase. a, Representative RT-qPCR data of Stra8, Rec8 and Sycp3 transcripts (normalized to Tbp $)$ in isolated PGCs. Error bars indicate + s.d. of 2 technical replicates. ${ }^{*} P<0.05(t-$ test). b, IF staining of Oct4 and Stra8 in $R n f 2^{+}$and $R n f 2^{\text {cko }}$ female gonads at E12.5 and E13.5. Scale bars: $10 \mu \mathrm{m}$. c, Classification of Cdh1-positive PGCs according to Rnf2 and Sycp3 protein levels in $R_{n f 2}{ }^{+}$and $R n f 2^{\text {cko }}$ E13.5 gonads of individual embryos. In brackets: number of PGCs scored per embryo. d, IF staining of Sycp3 and Rnf2 in $R_{n f 2}{ }^{+}$and $R n f 2^{c k o}$ E14.5 ovaries. Scale bars: $10 \mu \mathrm{m}$.

Figure 4 | PRC1 antagonizes RA signaling and maintains Stra8 in a repressive chromatin state. a, Representative RT-qPCR data of Rnf2, Stra8, Rec8 and Sycp3 transcripts (normalized to Tbp) in isolated PGCs. Error bars indicate +s.d. of 2-4 technical replicates. ${ }^{*} P<0.01 ;{ }^{* *} P<1.0 \mathrm{E}-3 ;{ }^{* * *} P<1.0 \mathrm{E}-4$ (t-test). b, IF staining of Oct4 and number of Oct4-positive PGCs in $R n f 2^{+}$and $R n f 2^{\text {cko }}$ whole gonads and mesonephroi from E12.5 female embryos, treated with WIN18446 or vehicle at E10.5. Graph shows mean with + s.d.. $n=5-6$. Scale bars: $300 \mu m$. c, $\mu$ ChIP analysis for $\mathrm{H} 3 \mathrm{~K} 4 \mathrm{me} 3, \mathrm{H} 2 \mathrm{~K} 27 \mathrm{me} 3$ and Rnf2 at promoter region of Stra8 in PGCs isolated from $\mathrm{E} 11.5,12.5,13.5$ and $14.5 \mathrm{Rnf}^{+}$gonads. Error bars indicate + s.d. of 1-3 biological and 2-4 technical replicates. ${ }^{*} P<0.05 ;{ }^{* *} P<0.001$ ( $t$-test). d. Model for transcriptional regulation of meiotic genes (e.g. Stra8) and pluripotency genes (e.g. Oct4) during sex differentiation of female PGCs.

\section{METHODS}


Mice and embryo collection. Rnf2 ${ }^{\text {cko }}$ mice with Rnf2 deficient PGCs were generated by combining a floxed Rnf2 $\left(R n f 2^{F}\right)$ allele ${ }^{14}$ with the TNAP-cre transgene allele as illustrated in Supplementary Fig. 3. Introduction of the Ring1 mutation ${ }^{17}$ is illustrated in Supplementary Fig. 6a. For PGC isolation, embryos were sired by fathers that were homozygous for the Oct4( $\triangle P E)$-GFP transgene. Mice were maintained on a mixed background of $129 / \mathrm{Sv}$ and C57BL/6J. Embryos were obtained by timed matings, by scoring noon of the day following mating as 0.5 embryonic day of development (E0.5). The genotype of embryos was determined by PCR as described previously ${ }^{14}$. Sex of gonads was determined by PCR for Ubex1 using primers: F: 5'TGGTCTGGACCCAAACGCTGTCCACA, R: 5'GGCAGCAGCCATCACATAATCCAGATG. All experiments were performed in accordance with the Swiss animal protection laws and institutional guidelines.

Antibodies. For immunofluorescence analyses, following primary and secondary antibodies were used: polyclonal anti-Oct4 (sc-8628, 1:150), monoclonal anti-Rnf2 (gift from $\mathrm{H}$. Koseki, 1:400) ${ }^{28}$, monoclonal anti-E-cadherin (Invitrogen, 1:250), polyclonal anti-Stra8 (rabbit, gift from M. Griswold, 1:1000) ${ }^{29}$, polyclonal anti-Sycp3 (rabbit, gift from C. Heyting, 1:500) ${ }^{30}$, polyclonal anti-Mel18 (sc-10774, 1:50), polyclonal anti-RYBP $(1: 400)^{31}$, monoclonal anti-H2AK119u1 (Cell signaling, 1:500), polyclonal antiH3K27me3 (gift from T. Jenuwein, 1:500) 32, monoclonal anti-Ezh2 (Novocastra, 1:200), anti-goat IgG-Alexa 488, anti-rabbit IgG-Alexa 488, anti-mouse IgG-Alexa 555, and anti-rat Cy5. For ChIP analysis, anti-H3K4me3 (Millipore, 17-614), anti-H3K27me3 (Millipore, 07-449) and anti-Rnf2 (Active motif, 39663) were used.

Immunofluorescence. For whole mount stainings, dissected gonads with mesonephroi were fixed for $15 \mathrm{~min}$ in $3 \%$ paraformaldehyde in PBS $(\mathrm{pH} \mathrm{7.4)}$ ) and permeabilized with $0.5 \%$ Triton-X100 in PBS for 20 min on ice. Fixed embryos were blocked overnight at $4^{\circ} \mathrm{C}$ in PBS containing $0.1 \%$ Triton-X100, 10\% BSA and 5\% normal donkey serum, and were then incubated with primary antibodies in blocking solution overnight at $4^{\circ} \mathrm{C}$. Gonads were washed three times for 1 hour in PBS 
containing $0.1 \%$ Triton-X 100 and $2 \%$ BSA before application of secondary antibodies. For detection, secondary antibodies were diluted 1:500 in blocking solution and gonads were incubated overnight at $4^{\circ} \mathrm{C}$ followed by three washing steps for 1 hour in PBS with $0.1 \%$ Triton-X 100. Gonads were stained briefly with DAPI and mounted in Vectashield (Vector). For gonadal section stainings, the posterior part of embryos or gonads with mesonephroi were frozen in Tissue-Tek O.C.T. ${ }^{\mathrm{TM}}$ compound (Sakura Finetek) on dry ice. Alternatively, the materials were fixed with $3 \%$ paraformaldehyde in PBS $(\mathrm{pH} 7.4)$ for 10 minutes, soaked in $30 \%$ sucrose solution overnight and embedded in O.C.T. compound. $12 \mu \mathrm{m}$ thick cryo-sections were cut from frozen blocks with Microm HM355S. Cryo-sections were fixed with $3 \%$ paraformaldehyde for 10 minutes at room temperature (RT), permeabilized in $0.5 \%$ Triton-X 100 in PBS for 4 minutes at $4^{\circ} \mathrm{C}$ and blocked for 30 minutes in PBS containing 1\% BSA at RT. Sections were incubated with primary antibodies in blocking solution overnight at $4^{\circ} \mathrm{C}$ and subsequently washed three times for 10 minutes in PBS with $0.05 \%$ Tween-20. Incubation of secondary antibodies was done in the blocking solution for 1 hour at RT.

Microscopy and image analysis. Immunofluorescence stainings of gonads were analyzed using the Zeiss LSM700 confocal microscope. For whole mount gonads, images were acquired by using a tile function with a z-series of $1 \mu \mathrm{m}$ slices in ZEN software and whole image was reconstructed using the XUV-tools software. We counted the number of Oct4-positive cells using a spot function in Imaris (Bitplane) software.

Isolation of PGCs expressing the Oct4( $\triangle P E)$-GFP transgene by FACS. Dissected gonads were enzymatically disrupted using $0.025 \%$ trypsin at $37^{\circ} \mathrm{C}$ for 8 minutes. Trypsin activity was inhibited by adding fetal calf serum in Hank's Buffer Salt Solution (HBSS) without Phenol Red. Gonads were dispersed by pipetting and subjected to Fluorescent Activated Cell Sorting. Embryos were processed individually for expression analysis. PGCs isolated from several embryos were pooled for ChIP analysis. 
Quantitative real-time PCR (RT-qPCR). Total RNA was extracted from isolated PGCs or surrounding somatic cells from individual embryos using the PicoPure ${ }^{T M}$ RNA Isolation Kit (KIT0202) according to the manufacturer's instructions (Stratagene) with the addition of $100 \mathrm{ng}$ E.Coli rRNA as carrier. Reverse transcription was performed using SuperScript III Reverse Transcriptase (Invitrogen) according to the manufacturer's protocol. qPCR reactions were performed with cDNA corresponding to 20 cells using the SYBR Green PCR Master Mix (Applied Biosystem) in an ABI Prism 7000 Real time PCR machine. All real time PCR measurements were normalized to the endogenous expression level of Tbp. We performed RT-qPCR analyses on multiple pairs of $R n f 2^{c k o}$ and control littermates. Data in figures present technical replicates for pairs of genotypes. Primers used:

Rnf2: F: 5'- TTAGAAGTGGCAACAAAGAGTG, R: 5'- CGCTTCATACTCATCACGAC, Oct4: F: 5'- GATGCTGTGAGCCAAGGCAAG, R: 5'- GGCTCCTGATCAACAGCATCAC, Nanog:F: 5'- CTTTCACCTATTAAGGTGCTTGC, R: 5'-

TGGCATCGGTTCATCATGGTAC,

Stra8: F: 5'- CAAAAGCCTTGGCTGTGTTA, R: 5'- AAAGGTCTCCAGGCACTTCA, Rec8: F: 5'- CCAACAAGGAGCTGGACTTC, R: 5'- GGACAGCACCAAGAGCAGAT, Sycp3: F: 5'- GTGTTGCAGCAGTGGGAAC, R: 5'- GCTTTCATTCTCTGGCTCTGA, Aldh1a2: F: 5'- CCCTGACAGTGGCTTTGAGT, R: 5'- CTGTGGGTTGAAGGGAGCTA, Crabp1: F: 5'- GCTTCGAGGAGGAGACAGTG, R: 5'- CAGCTCTCGGGTCCAGTAAG, Crabp2: F: 5'- GCCGAGAACTGACCAATGAT, R: 5'- GGAAGTCGTCTCAGGCAGTT, Tbp: F: 5'- TGCTGTTGGTGATTGTTGGT, R: 5'- AACTGGCTTGTGTGGGAAAG.

Expression profiling of PGCs and data analysis. We performed expression profiling on PGCs isolated from 3 pairs of $R n f 2^{\text {cko }}$ and control littermates for each developmental time point. RNA was extracted from 500 PGCs isolated per embryo using the PicoPure ${ }^{\mathrm{TM}}$ RNA Isolation Kit (KIT0202) according to the manufacturer's instructions (Stratagene). The quality of the RNA was assessed using the Agilent 2100 Bioanalyzer and RNA 6000 Pico Chip. The extracted RNA was converted into OmniPlex WTA 
cDNA libraries and amplified by WTA PCR using reagents supplied with the TransPlex Whole Transcriptome Amplification kit (WTA1, Sigma, USA) following the manufacturer's instructions with minor modifications. The obtained cDNA was purified using the GeneChip cDNA Sample Cleanup Module (Affymetrix). The labeling, fragmentation and hybridization of cDNA was performed according to Affymetrix instructions (GeneChip Whole Transcription Sense Target Labeling technical manual, Rev. 2) with minor modifications. Samples were hybridized to Affymetrx Mouse Gene 1.0 arrays. Microarray quality control and analysis was carried out in R 2.10.0 and Bioconductor 2.5. Briefly, array quality was assessed using the "arrayQualityMetrics" package. Raw data was read into R and normalized with RMA using the "affy" package and differentially expressed genes were identified using the empirical Bayes method ( $F$ test) implemented in the LIMMA package. $P$-values were adjusted for FDR using the Benjamini and Hochberg correction. Probe sets with a log2 average contrast signal of at least 3 , an adjusted $P$-value of $<0.05$, and an absolute linear fold-change of at least 1.5-fold were selected. The $P$-values reported for enriched GO terms were obtained using GO Stat (http://gostat.wehi.edu.au) (Supplementary Figure 7, 8, Supplementary Table 2). List of genes which belong to the GO-term "meiosis" (GO:0007126) were obtained using $\mathrm{R}$ annotation packages (library(org.Mm.eg.db) and library(GO.db)) (Figure 2d, Supplementary Table 1). For RNA sequencing experiments, RNA was extracted from 500 PGCs per embryo using RNeasy Micro Kit (QIAGEN). The RNA amplification and cDNA generation were performed using NuGEN Ovation RNA-seq System V2 (Part No. 7102) and sequencing libraries were prepared using Truseq DNA Sample Preparation Kit (low-throughput protocol) (Part\# 15005180 Rev. C). Barcoded libraries were sequenced in one lane of Illumina Hiseq instrument. The resulting sequencing reads were filtered, aligned to Refseq gene models and weighted as described previously ${ }^{33}$.

Treatment of gonads with agonist and antagonist of RA signaling. Both gonads per embryo were dissected and were cultured together or separately in a drop of 
Dulbecco's Minimal Eagle Medium (DMEM) supplemented with $10 \%$ fetal calf serum at $37^{\circ} \mathrm{C}$ in a humidified atmosphere of $5 \% \mathrm{CO}_{2}$ in air. All-trans RA (Sigma) and WIN18446 (N,N'-Octmethylenebis(dichloroacetamide), ABCR) were dissolved in DMSO. These compounds were added to culture media with concentration of $0.5 \mu \mathrm{M}$ for all-trans RA or $2 \mu \mathrm{M}$ for WIN18446. Control cultures were treated with DMSO vehicle as appropriate. For in vivo administration of WIN18446, $100 \mathrm{mg} / \mathrm{ml}$ stock solution (in DMSO) was diluted with oil and $2.5 \mathrm{mg}$ was injected intra-peritoneally into pregnant female mice at E10.5. Embryos were collected at E12.5. DMSO mixed with oil was injected into pregnant female mice for control experiments.

STA (Specific Target Amplification) RT-qPCR on single GV oocytes. GV oocytes were isolated from ovaries and carefully washed with removing cumulus cells. STA was performed using the CellsDirect ${ }^{\top \mathrm{M}}$ One-Step qRT-PCR Kit (11753-100) according to the manufacturer's instructions (Invitrogen). Briefly, the oocytes were individually added to the reaction mix containing RNase Inhibitor (Ambion) and primers of target genes of interest. After amplification, the samples were treated with Exonuclease I (New England Biolabs) and used as template for qPCR. Primers used:

Rnf2: F: 5'- CAGGCCCCATCCAACTCTTA, R: 5'- CAACAGTGGCATTGCCTGAA;

Ssu72:F: 5'- GGTGTGCTCGAGTAACCAGAA, R: CAAAGGAGCGGACACTGAAAC.

Micro Chromatin Immunoprecipitation (uChIP) analysis. Small scale ChIP experiment was performed as previously described ${ }^{27}$ with some modifications. Briefly, 15'000 FACS sorted PGCs were cross-linked with $0.5 \%$ paraformaldehyde in PBS for $10 \mathrm{~min}$ at room temperature and quenched with $125 \mathrm{mM}$ glycine. PGCs were then lysed in $50 \mathrm{mM}$ Tris- $\mathrm{HCl}(\mathrm{pH} 8.0), 10 \mathrm{mM}$ EDTA, $1 \% \mathrm{SDS}$, and protease inhibitors. The cell lysate was sonicated in a Diagenode Bioruptor to achieve a mean DNA fragment size around 200-400 base pairs. After centrifugation, the supernatants were diluted with RIPA buffer to an equivalent of 2'500 PGCs and incubated with antibody-bound protein G-magnetic beads overnight at $4{ }^{\circ} \mathrm{C}$. The beads were washed four times with the RIPA 
buffer and one time with TE buffer, and bead-bound complexes were incubated with complete elution buffer (20mM Tris- $\mathrm{HCl}$ (pH7.5), $5 \mathrm{mM}$ EDTA, $50 \mathrm{mM} \mathrm{NaCl}, 1 \%$ SDS, $50 \mathrm{mg} / \mathrm{ml}$ proteinase $\mathrm{K}$ ) at $68^{\circ} \mathrm{C}$ for DNA elution, cross-link reversal and protein digestion. Finally, IP-ed DNA was purified by phenol-chloroform extraction and ethanol precipitation and dissolved in MilliQ water for quantitative real-time PCR analysis. Primers used:

Stra8: F: 5'- GTATCGCCGTAACTCCCAGA, R: 5'- GCAGATGACCCTCACACAAG; HoxA9: F: 5'- GGAGGGAGGGGAGTAACAAA, R: 5'- TCACCTCGCCTAGTTTCTGG.

28 Atsuta, T. et al. Production of monoclonal antibodies against mammalian Ring1B proteins. Hybridoma 20, 43-46 (2001).

29 Hogarth, C. A. et al. Turning a Spermatogenic Wave into a Tsunami: Synchronizing Murine Spermatogenesis Using WIN 18,446. Biology of reproduction (2013).

30 Lammers, J. H. et al. The gene encoding a major component of the lateral elements of synaptonemal complexes of the rat is related to X-linked lymphocyteregulated genes. Molecular and cellular biology 14, 1137-1146 (1994).

31 Garcia, E., Marcos-Gutierrez, C., del Mar Lorente, M., Moreno, J. C. \& Vidal, M. RYBP, a new repressor protein that interacts with components of the mammalian Polycomb complex, and with the transcription factor YY1. Embo $J$ 18, 3404-3418 (1999).

32 Peters, A. H. et al. Partitioning and plasticity of repressive histone methylation states in mammalian chromatin. Mol Cell 12, 1577-1589 (2003).

33 Tippmann, S. C. et al. Chromatin measurements reveal contributions of synthesis and decay to steady-state mRNA levels. Mol Syst Biol 8, 593 (2012). 

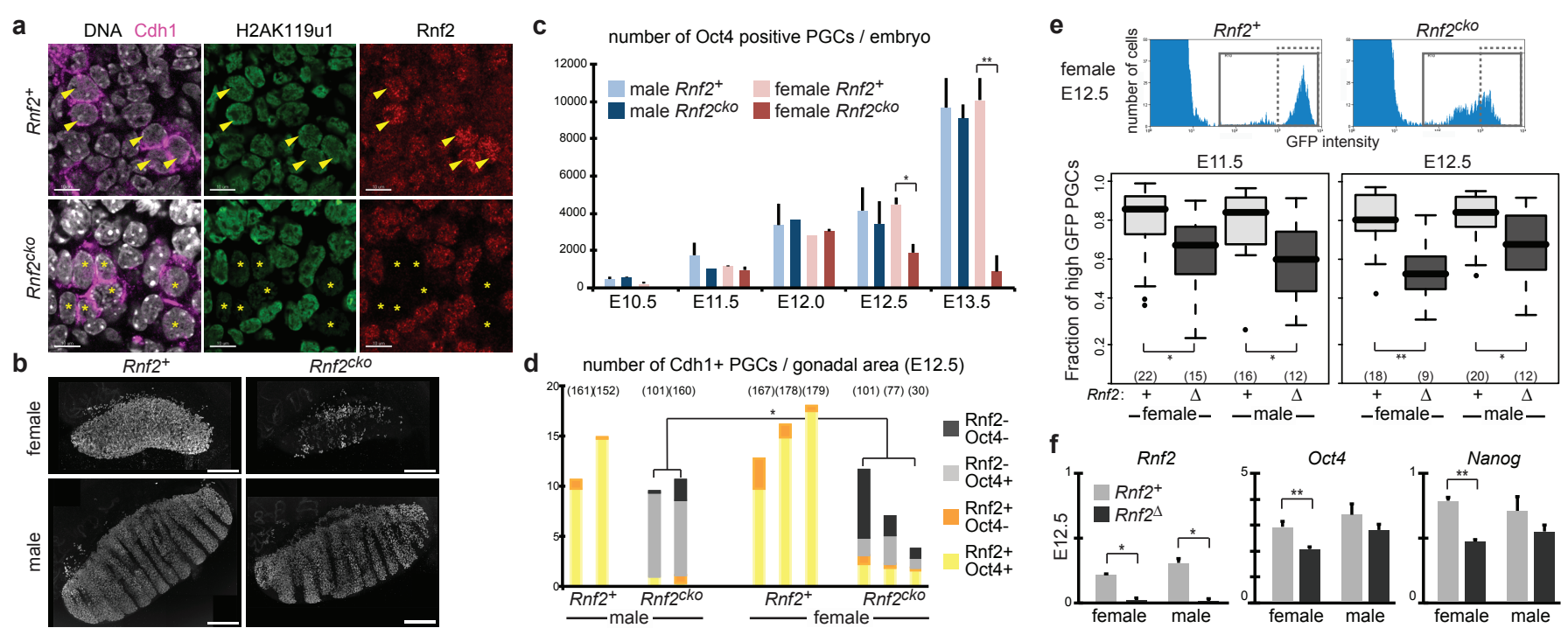


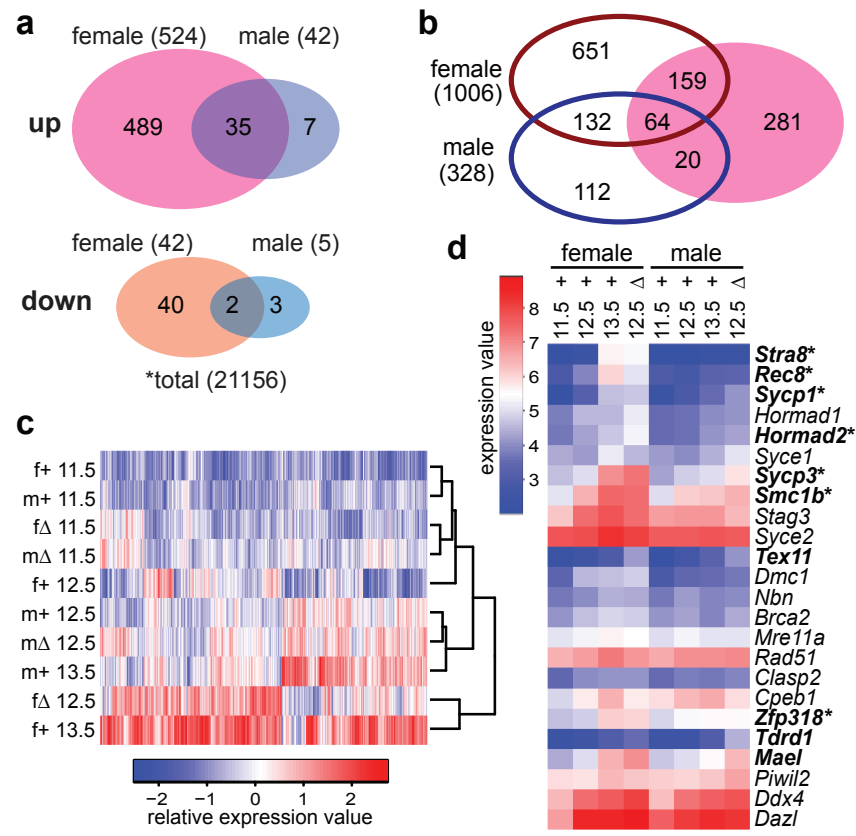

Yokobayashi et al

Figure 2 


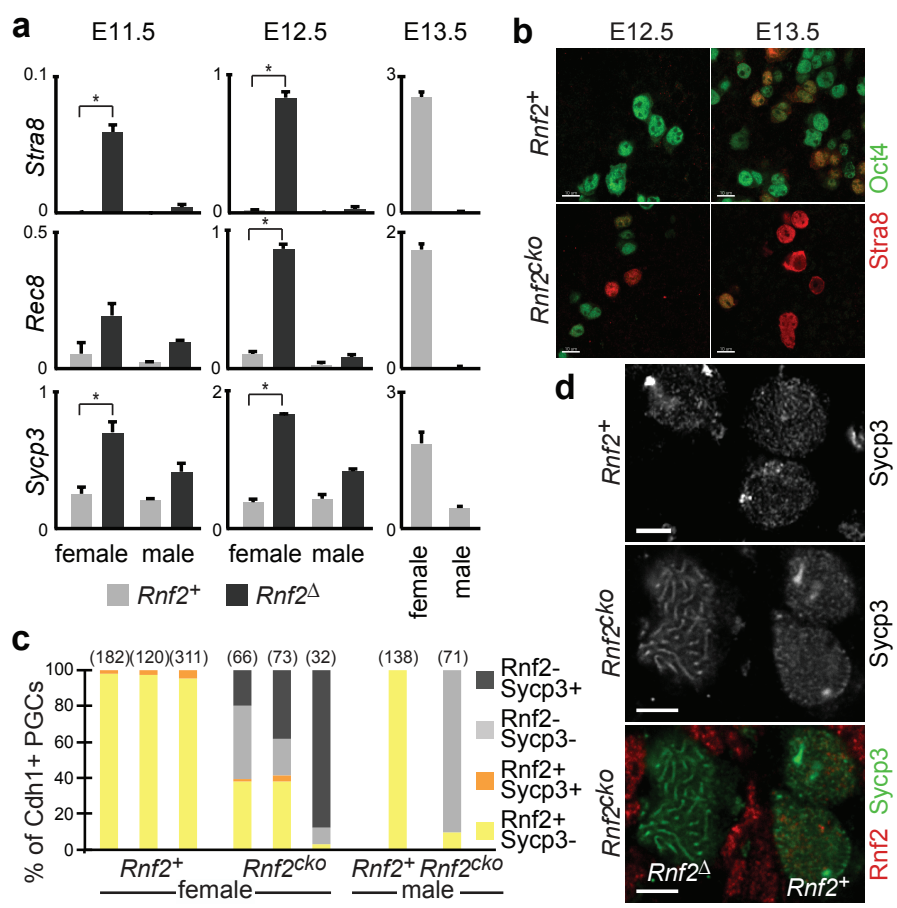

Yokobayashi et al.

Figure 3 


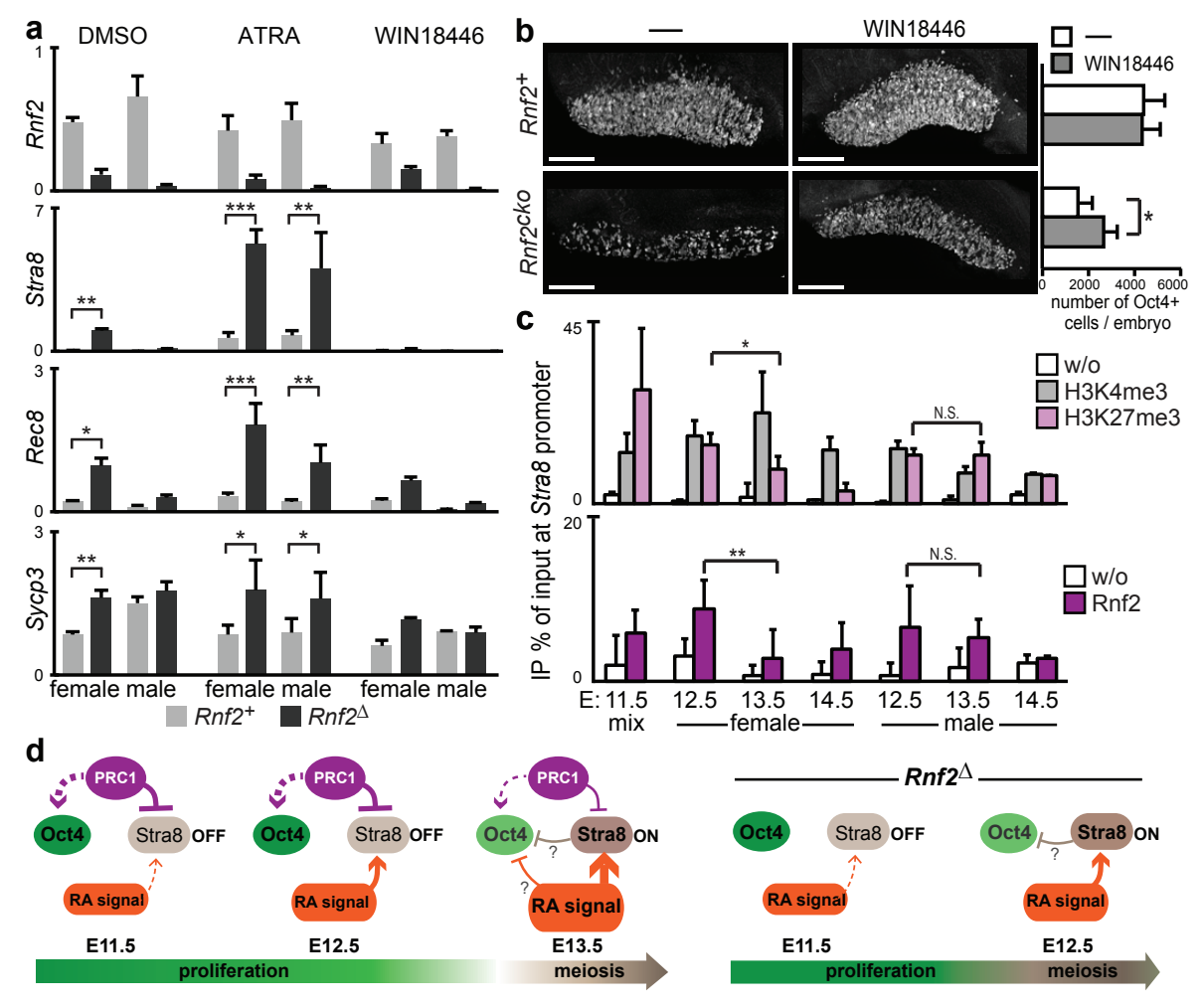

Yokobayashi et al.

Figure 4 